Moreover, the tumorigenicity was abolished by PMN-MDSC depletion. Notably, intrasplenic injection of a mouse melanoma cell line B16F10 exhibited an increased level of liver-infiltrating PMN-MDSCs and enhanced liver metastasis in male TG mice compared to control mice. Moreover, depletion of PMN-MDSCs suppressed metastasis in liver. Mechanistically, anti-tumor NKT cells, rather than NK cells and $\mathrm{CD} 8^{+} \mathrm{T}$ cells, were negatively correlated with tumor weight and MDSC proportion, indicating involvement of cross-talk between MDSC and NKT in liver metastasis.

Conclusions Our findings suggest that hepatic CCRK expression create a tumor growth- and metastasis-supportive liver microenvironment via enhancing immunosuppression.

\section{IDDF2019-ABS-0266 EFFECTS OF THYROID HORMONE TREATMENT ON HEPATIC GLUCOSE PRODUCTION AND RENAL REABSORPTION OF GLUCOSE IN ALLOXAN-INDUCED DIABETIC WISTAR RATS}

Ranbir Singh*. Department of Applied Sciences, Raja Mahendra Pratap Post Graduate College, Haridwar (Uttarakhand), India

\subsection{6/gutjnl-2019-IDDFabstracts. 106}

Background The thyroid hormone (TH) plays an important role in glucose metabolism. Recently, we showed that the TH improves glycemia control by decreasing cytokines expression in the adipose tissue and skeletal muscle of alloxan-induced diabetic rats, which were also shown to present primary hypothyroidism. In this context, this study aims to investigate whether the chronic treatment of diabetic rats with T3 could affect other tissues that are involved in the control of glucose homeostasis, as the liver and kidney.

Methods Adult male Wistar rats were divided into nondiabetic, diabetic, and diabetic treated with T3 $(1.5 \mu \mathrm{g} / 100 \mathrm{~g}$ BW for 4 weeks). Diabetes was induced by alloxan monohydrate $(150 \mathrm{mg} / \mathrm{kg}, \mathrm{BW}$, i.p.). Animals showing fasting blood glucose levels greater than $250 \mathrm{mg} / \mathrm{dL}$ were selected for the study.

Results After treatment, we measured the blood glucose, serum T3, T4, TSH, and insulin concentration, hepatic glucose production by liver perfusion, liver PEPCK, GAPDH, and pAKT expression, as well as urine glucose concentration and renal expression of SGLT2 and GLUT2. T3 reduced blood glucose, hepatic glucose production, liver PEPCK, GAPDH, and pAKT content and the renal expression of SGLT2 and increased glycosuria.

Conclusions Results suggest that the decreased hepatic glucose output and increased glucose excretion induced by T3 treatment are important mechanisms that contribute to reducing serum concentration of glucose, accounting for the improvement of glucose homeostasis control in diabetic rats.

\section{IDDF2019-ABS-0267 EPIDEMIOLOGICAL STUDY OF THE PREVALENCE OF HEPATITIS B VIRUS IN SCHOOL-AGE CHILDREN IN WEST INDIAN STATE}

Tanvi Sharma*, Vijay Sharma. Regional Institute of Health Medicine and Research, Jaipur, Rajasthan, India

10.1136/gutjnl-2019-IDDFabstracts. 107
Background In India, chronic hepatitis B is acquired predominantly by horizontal transmission in early childhood and to a lesser extent by perinatal transmission. The exact mode of horizontal transmission remains undefined, may be close contact, body fluid contact. The age of acquisition of HBV is an important determinant of outcome; (e.g., $>90 \%$ in newborns (vertical transmission), 30\% in children aged 2-5 years and $<5 \%$ in adults). HBV also spread by parenteral transmission at any age including intravenous drug use, unsafe therapeutic injections, occupational injuries or nosocomial transmission. There is no data of HBV prevalence among school children in the western Indian state of Rajasthan so we decided to conduct an epidemiological study in the year 2018-2019.

Methods Senior liver expert designed and planned the study, school administration, social workers and school student volunteers were involved in arranging HBV test camps at government schools. Discussion, presentations, study material distribution, coordination, data collection was done by students under the supervision of team, five government school in Jaipur district were covered, HBV ELISA spot test was done.

Results Total 1255 students were screened, out of them 17 were positive but asymptomatic, 11 male, 6 female, age 10 to 16 years. The study suggests a lower prevalence of $\mathrm{HBV}$ in west India likely due to high vaccination coverage. Old studies suggest rates of HBsAg-positivity 2.14-2.25\% among children $<5$ years of age and $4.3-7.2 \%$ among the entire paediatric population, while $6.7 \%$ among those with liver disease, $2 / 3 \mathrm{rd}$ $\mathrm{HBeAg}$ negative. Though horizontal transmission is the predominant mode, the contribution of vertical transmission is also important. Hepatitis B is a significant public health problem in India, yet disease awareness among Indian people, the key to decreasing disease burden is dismally low. The majority of disease cases progress silently and patients present in advanced stages when decompensated CLD or HCC has already developed.

Conclusions Prevalence of $\mathrm{HBV}$ is $1.35 \%$ in west Indian school kids, countrywide vaccination coverage should be more aggressive in the pediatric population. Our emphasis should be on health education of general and high-risk populations along with aggressive vaccination strategies especially for tribals and high-risk groups.

\section{IDDF2019-ABS-0302 IDENTIFICATION OF DNA METHYLATION SIGNATURES FOR MICROVASCULAR INVASION IN HEPATOCELLULAR CARCINOMA}

Lixia Xu*, Sui Pen, Qiye He, Zhihang Chen, Ming Kuang. The First Affiliated Hospital, Sun Yat-sen University, China

\subsection{6/gutjnl-2019-IDDFabstracts. 108}

Background The presence of microvascular invasion (MVI) reduces overall survival of hepatocellular carcinoma (HCC). Recent studies showed DNA methylation markers could be applied to the diagnosis of cancers. However, it is unclear whether DNA methylation signatures could help diagnose $\underline{\mathrm{MVI}}$ in HCC.

Methods To identify DNA methylation markers for HCC and MVI diagnosis, we first generated genome-wide DNA methylation profiles from HCC tissues and adjacent normal liver 
tissues of 37 HCC patients (20 MVI+ and 17 MVI-). We also investigated DNA methylation profiles of plasma samples from these 37 HCC patients and 30 normal individuals as controls to minimize the interference of random background DNA methylation signals. Tissue and plasma samples were prepared into DNA methylation library and sequenced on Illumina Hiseq X10 platform. Using methylation haplotype load (MHL) and unmethylation haplotype load (UMHL) as metrics, we quantified DNA methylation profiles on methylation haplotype blocks (MHBs) by computing the degree of linkage between methylated or unmethylated CpGs in HCC and adjacent normal samples to identify discriminatory markers. Grouping samples from our cohort into training and validation sets respectively, we employed two supervised machine learning algorithms, random forest (RF) and support vector machine (SVM) to train and cross-validate binary predictive models.

Results Supervised analyses identified 65 MHBs as classifiers for HCC tissues and adjacent normal liver tissues. Both RFand SVM-built models were highly accurate in classifying HCC and normal liver tissues with AUC no less than 0.98 (AUC: 98\%, CI: 97.3\% 98.8\% for RF model; AUC: 99.9\%, CI: $99.9 \sim 99.9 \%$ for SVM model). We applied the RFtrained classification model to differentiate HCC plasma DNA from those of healthy controls, with AUC of 96\% (CI: $95.1 \%$ - 96.9\%). We further identified 6 MHL-quantified MHBs and 5 UMHL-quantified MHBs as classifiers for MVIand MVI+ samples. Combining the MHBs' methylation scores as classifiers and applying RF method, we trained and cross-validated MVI- and MVI+ classification models. We found the AUC of these models is $85.9 \%$ (CI: $83.5 \%$ $88.3 \%)$.

Conclusions These data showed that DNA methylation signatures can provide outstanding diagnostic accuracy for HCC and MVI.

\section{IDDF2019-ABS-0320 TERMINALIA ARJUNA BARK EXTRACT PROTECTS AGAINST EARLY ALCOHOL- INDUCED LIVER INJURY IN THE RAT}

Ramesh Kumar Meena*, Tejpal Singh. Department of Biological Sciences, University of Rajasthan, Jaipur, India

\subsection{6/gutjnl-2019-IDDFabstracts.109}

Background Terminalia arjuna, an indigenous plant used in ayurvedic medicine in India, primarily as a cardiotonic is also used in treating diabetes, anemia, tumors and hypertension. Oxidants have been shown to be involved in the alcoholinduced liver injury. Arjunolic acid (AA) is an oleanane triterpenoid found mainly in the heartwood of Terminalia arjuna. This study was designed to determine whether Terminalia arjuna bark (TAB) extract, composed mostly of ellagitannins oligomers, protects against early alcohol-induced liver injury in rats.

Methods Total fifty-eight male Wistar rats were fed high-fat liquid diets with or without ethanol (10-14 g/kg per day) and $\mathrm{TAB}$ extract $(300 \mathrm{mg} / \mathrm{kg}$ per day) continuously for $\underline{4}$ weeks using an enteral feeding protocol.

Results Mean body weight gains (approximately $4 \mathrm{~g} /$ day) were not significantly different between treatment groups. TAB extract did not affect average daily urine ethanol concentrations (approximately $200 \mathrm{mg} / \mathrm{dL}$ ). After $\underline{4}$ weeks, serum alanine amino transferase levels of the ethanol group were increased nearly fourfold $(110 \pm 16 \mathrm{IU} / \mathrm{L})$ compared to control values $(35 \pm 3 \mathrm{IU} / \mathrm{L})$; this effect of ethanol was blocked by $\mathrm{TAB}$ extract $(60 \pm 6 \mathrm{IU} / \mathrm{L})$. Additionally, enteral ethanol caused severe fat accumulation, mild inflammation, and necrosis in the liver; TAB extract significantly blunted these changes. Increases in liver TNFalpha protein levels caused by ethanol were completely blocked by TAB extract. Further, ethanol significantly increased the accumulation of protein adducts of 4hydroxynonenal, a product of lipid peroxidation serving as an index of oxidative stress; again this was counteracted by the addition of TAB extract.

Conclusions The result indicates that the TAB extracts exhibit the antioxidant activity through correction of oxidative stress and validates the traditional use of Terminalia arjuna to prevents early alcohol-induced liver injury.

\section{IDDF2019-ABS-0325 SUPERIOR EFFICACY AND LONG-TERM SURVIVAL BENEFIT OF HDAC8 AND PD-L1 CO-BLOCKADE IN LIVER CANCER IMMUNOTHERAPY}

${ }^{1}$ Weiqin Yang*, Yu Feng, ${ }^{1}$ Jingying Zhou, ${ }^{1}$ Otto KW Cheung, ${ }^{2}$ Hanyong Sun, ${ }^{3}$ Stephen L Chan, ${ }^{4}$ Anthony WH Chan, ${ }^{5}$ Zhiwei Chen, ${ }^{6}$ Kevin YL Yip, ${ }^{4}$ Ka-Fai To, ${ }^{2}$ Joseph JY Sung, ${ }^{1}$ Alfred SL Cheng. 'School of Biomedical Sciences, The Chinese University of Hong Kong, Hong Kong; ${ }^{2}$ Department of Medicine and Therapeutics, The Chinese University of Hong Kong, Hong Kong; ${ }^{3}$ Department of Clinical Oncology, The Chinese University of Hong Kong, Hong Kong: ${ }^{4}$ Department of Anatomical and Cellular Pathology, The Chinese University of Hong Kong, Hong Kong; ${ }^{5}$ AIDS Institute, The University of Hong Kong, Hong Kong; ${ }^{6}$ Department of Computer Science Engineering, The Chinese University of Hong Kong, Hong Kong

\subsection{6/gutjnl-2019-IDDFabstracts.110}

Background The heterogeneous responses to immune-checkpoint blockade (ICB) therapy e.g. anti-programmed deathligand 1 (PD-L1) antibody are attributable to the complex interplay between a range of cancer-cell-autonomous cues and immunosuppressive tumor microenvironment. Two recent phase I/II trials of $\mathrm{PD}-1$ checkpoint inhibitors in patients with advanced hepatocellular carcinoma (HCC) have produced promising results, yet the objective response rates were relatively low $(<20 \%)$. Accumulating evidence underscore the fundamental importance of epigenetic regulation in tumor immune evasion. We have previously elucidated a critical role of histone deacetylase 8 (HDAC8) in hepatic carcinogenesis (Cancer Research 2015;75:4803-16). Here, we aim to investigate the therapeutic potential of a HDAC8-specific inhibitor PCI-34051 in preclinical HCC models.

Methods The effect of PCI-34051 on tumorigenicity was investigated in orthotopic HCC mouse models. Immune profiling in tumor microenvironment was determined by multi-color flow cytometry. The underlying mechanism was investigated by integrative epigenomics analysis. The anti-tumor efficacy of combined therapy with PCI-34051 and anti-PD-L1 antibody was further determined.

Results PCI-34051 significantly suppressed tumorigenicity in immunocompetent but not immunodeficient mice, which was accompanied by increased effector $\mathrm{T}$ cell tumor infiltration. Mechanistically, tumor-intrinsic HDAC8 epigenetically silenced chemokine CCL4 expression via H3K27 deacetylation at the enhancer to inhibit $\mathrm{T}$ cell infiltration to tumor. Furthermore, PCI-34051 dramatically improved the therapeutic efficacy of 